These substances do not pass through the cell or nuclear membranes, but the nuclear membrane is often pushed out toward the anode by the pressure of the (basic-staining) chromatin.

During the process of mitosis, however, the above rules do not hold. As the process of mitosis advances, more current is required to move the chromatin, and more acid-staining proteid is carried along with it. After the mitotic spindle is formed, it (including the chromosomes) is carried toward the anode without alternation of its axial orientation.

These facts disprove the hypothesis that the chromosomes are drawn to the poles of the spindle by electrostatic stress, the poles and chromosomes being of opposite sign, for if this were true, the poles of the spindle would not move toward the anode with the chromosomes, but would move toward the cathode.

It is probable that the mitotic spindle consists chiefly of proteids in the "gel" phase, and that this gelation prevents the separation of acidophile and basophile substances by the current. That the cells were not killed and the spindles coagulated, during the experiments, is demonstrated by the fact that a cell in which the spindle was displaced by the current divided. In this case the cytoplasm was divided unequally, as the division passed through the equator of the displaced spindle.

$$
70(480)
$$

\title{
Nitrogen and sulphur metabolism in morbus ceruleus.
}

\section{By N. B. FOSTER.}

In morbus ceruleus due to congenital cardiac defect there are found two conditions which suggest that in this disease the tissues are not so adequately furnished with oxygen as in health. These conditions are the abnormally high carbon dioxide content of the venous blood (Lepine) and the erythrocytosis, the latter being a direct response of the hemopoietic tissues to a lowered oxygen tension (Seller). It would seem plausible that in some severe cases the metabolism might be altered by the conditions mentioned. The case observed in this study was a child that presented all the features of morbus ceruleus due to congenital cardiac defect. Analyses of the urine failed to show anything abnormal in the nitrogen partition. The comparison of the amounts of 
neutral sulphur, wherein deficient oxidation would be most apt to be manifested, with the amounts of a normal child, shows very slightly higher figures but the difference is not sufficient to justify the conclusion of deficient oxidation.

\section{I $(48 \mathrm{I})$ \\ On parenteral protein assimilation. \\ By P. A. LeVENe and G. M. MEYeR.}

[From the Department of Chemistry of the Laboratories of the Rockefeller Institute for Medical Research.]

The results of recent work on the fate of protein introduced parenterally led to the conviction that such protein is assimilated and utilized by the organism in the same manner and in accordance with the same laws as protein ingested per os. However, there exists a considerable divergence in the views on the mechanism by which this assimilation is accomplished.

Very recently Freund advanced a theory that protein introduced into the organism subcutaneously or through the circulation is eliminated into the intestinal tract where it undergoes the usual digestion and absorption.

The present investigation aimed to test the correctness of the last theory. An animal deprived of its jejunum and ileum was placed on a standard diet and brought into a condition of nitrogenous equilibrium. On the days of experiments the animal received a subcutaneous injection of horse serum, heated for one half hour at $60^{\circ} \mathrm{C}$. The volume of injected serum was equivalent to I. 5 grams of nitrogen. The elimination of additional nitrogen was followed for several days following the days of the serum injection. In all experiments was noted a complete retention of the protein introduced parenterally in the same manner as this occurs in normal animals. Thus the theory of Freund is contradicted by the results of our experiments.

$$
72(482)
$$

A method of isolating the cerebro-medullary circulation.

By ARTHUR BRADLEY EISENBREY.

[From the Carnegie Laboratory of New York University.]

In a recent study by Dr. R. M. Pearce and myself of the mechanism of certain experimental conditions of low blood pressure, the following difficulties were encountered: 